\title{
Fair Allocations of a Renewable Resource
}

\author{
JOEL SOBEL \\ University of California-San Diego, La Jolla, California 92093 \\ Received January 23, 1978; revised April 10, 1979
}

\begin{abstract}
In an economy with finitely many agents, one renewable resource, and an infinite horizon, it is shown that there is exactly one maximal allocation corresponding to given limiting shares of consumption and this allocation converges monotonically. Therefore, if there is no discounting, at most one fair maximal program exists--that which gives an equal amount to each individual in the limit. In this allocation, envy is always finite. However, only in special cases is it envyfree. This is in contrast to the case of finite economies where envy-free and Pareto efficient allocations may not exist or, if they exist, may not be unique.
\end{abstract}

\section{INTRODUCTION}

Levhari and Mirman [5] consider an infinite horizon economy with a single renewable resource. In [5], two countries fish in a common ocean. The fish population reproduces in accordance with the usual neoclassical production function. Each country has a utility function, and there is a discount factor common to both. It is shown that the Cournot-Nash noncooperative duopoly equilibrium is in general not Pareto optimal.

In this paper the cooperative solution for the same model with a finite number of agents is considered. We seek consumption programs that are maximal and satisfy some fairness criterion. The main result is that any maximal program is globally asymptotically stable in that the value of capital stock (fish population) monotonically approaches the "golden-rule" value $\bar{x}$ (that is, $f^{\prime}(\bar{x})=1 / \beta$, where $f$ is the production function and $\beta$ the discount rate) and the consumption of the $i$ th agent monotonically approaches some fixed value $\theta_{i} \bar{c}$, where $\theta_{i} \geqslant 0, \sum_{i=1}^{n} \theta_{i}=1$ (here $\bar{c}=f(\bar{x})-\bar{x}$ is the "golden rule" consumption, and $n$ is the number of agents). Conversely, there is exactly one maximal program corresponding to any distribution of limiting consumption. Fairness then consists in a reasonable choice of limiting consumptions. If the agents are thought of as individuals, equal limiting consumptions would seem appropriate. If they represent countries, the limiting shares could be chosen proportional to population. In this way each individual could receive an equal limiting share of consumption. The allocations characterized by these definitions of fairness are not in general envy-free. 
However, in the undiscounted case $(\beta=1)$ it is shown that our definition of fairness is the only one that guarantees that envy will be finite, that is, the utility an agent could receive from someone else's consumption stream can exceed the utility he actually receives by at most a finite amount.

The second and third sections of this paper characterize the maximal states of a one-sector growth model. The model differs from the standard models in this area since there are many agents (countries). For the proof of existence, our model can be reduced to a case that requires the maximization of a single social utility function -an appropriate positive linear combination of the individual utlities. This is possible since any maximal allocation for the single function is maximal for the entire economy. The existence theorem of Gale [4] or Brock [1] then may be applied. However, this type of argument cannot be used to answer the uniqueness question. In the undiscounted case, there is no guarantee that a maximal allocation for the economy is maximal with respect to any weighted average of the agents' utility functions. (The reason for this is, essentially, that given any sequence $\left\{a_{t}^{i}\right\}, t=1,2, \ldots$, $i=1, \ldots, n, \lim \inf _{t \rightarrow \infty} \sum_{i=1}^{n} a_{t}{ }^{i} \geqslant \sum_{i=1}^{n} \lim _{\inf _{t \rightarrow \infty}} a_{t}{ }^{i}$ and the inequality may be strict.) Thus the identification of maximal programs with limiting consumptions, the object of Section 2, must be carried out directly. Although a literature on the uniqueness of optimal paths is available (the one-sector case is covered in [3]; a more general treatment is offered in [2]), it can only be applied to assert uniqueness of maximal programs that that result from maximizing a single function. Taken together, Sections 2 and 3 show that every efficient program can be viewed as a maximal program derived from a single function.

The existence of a fair efficient allocation is not in contrast to analogous finite models. For example, any competitive equilibrium for a pure exchange economy in which every agent is assigned an equal share of the initial resources is Pareto optimal and envy-free. Indeed, if the discount factor is less than one, and the model is treated as a private ownership economy with agents sharing profits equally, then any competitive equilibrium is efficient and envy-free. This is true only because there is no labor input in the production process. In more general economies with production fair and efficient allocations need not exist (an example is given in [8]).

It is normally not the case that the fair efficient allocation is unique. There is no reason to expect a unique competitive allocation in an equal income economy. Thus, what is special about the model presented here is that the equitable allocation is completely determined by the requirements of maximality and finite envy,

The reason for the distinction between the discounted and undiscounted cases is that the competitive equilibrum problem is well posed only when $\beta<1$. Since output remains bounded, the discounted sum of individual utilities is finite, and it is not difficult to modify standard arguments to show 
existence and optimality of competitive allocations when each agent receives a share of the profits from the (fish) production process. Thus the infinite horizon economy with $\beta<1$ behaves essentially like a finite economy.

When there is no discounting the situation is altered considerably. The fact that there is no envy-free maximal allocation means that the equal shares competitive allocation does not exist. Since utility and profits are unbounded, this fact is not surprising. However, the positive result - the existence of a unique maximal allocation with finite envy-is satisfactory. If we interpret the undiscounted infinite horizon model as an idealization of finite horizon models we can assert that all efficient envy-free allocations for finite models (having sufficient periods) yield nearly equal final period consumption.

\section{Definitions and Notation}

There are $n$ agents. Each agent $i$ has a utility function for consumption, $u_{i}:(0, \infty) \rightarrow \mathbb{R} . u_{i}$ is assumed to be strictly increasing, strictly concave, and twice continuously differentiable. Denote $\lim _{c \rightarrow 0} u_{i}(c)$ and $\lim _{c \rightarrow 0} u_{i}^{\prime}(c)$ by $u_{i}(0)$ and $u_{i}^{\prime}(0)$, respectively. These values need not be finite. A discount factor $\beta \in(0,1]$ is common to all consumers.

The technology is described by a twice continuously differentiable function $f:[0, \infty) \rightarrow[0, \infty)$ with $f(0)=0$. We assume that $f^{\prime}(x)>0>f^{\prime \prime}(x)$ for all $x$. We also assume that $f^{\prime}(x)>1 / \beta$ for some $x>0$, and that $f(\hat{x})=\hat{x}$ for some $\hat{x}>0$. It then follows that there is a unique $\bar{x} \in(0, \hat{x})$ satisfying $f^{\prime}(\bar{x})=1 / \beta$. Let $\bar{c}=f(\bar{x})-\bar{x}$. Notice $\bar{c}>\beta f(x)-x$ whenever $x \neq \bar{x}$.

A program is a sequence $\left\{\left(x_{t} ; \gamma_{t}\right)\right\}_{t=1}^{\infty}$ with $x_{t} \geqslant 0, \gamma_{t}=\left(c_{t}^{1}, \ldots, c_{t}^{n}\right)$, $c_{t}{ }^{i} \geqslant 0$ for all $i$ and $t$. Let $c_{t}=\sum_{i=1}^{n} c_{t}{ }^{i}$. A program $\left\{\left(x_{t} ; \gamma_{t}\right)\right\}$ is called feasible if, for some $x_{0}>0, c_{t}=f\left(x_{t-1}\right)-x_{t}$ for $t \geqslant 1$. We assume throughout that all programs start from a fixed $x_{0}>0$.

A sequence $\left\{\bar{c}_{t}^{i}\right\}_{t=1}^{\infty}$ is said to catch $u p$ to $\left\{c_{t}^{i}\right\}_{t=1}^{\infty}$ for agent $i$ if $\lim _{i n f} \inf _{T \rightarrow \infty} \times$ $\sum_{t=1}^{T} \beta^{t-1}\left[u_{i}\left(\bar{c}_{t}{ }^{i}\right)-u_{i}\left(c_{t}{ }^{i}\right)\right] \geqslant 0$. A program $\left\{\left(\bar{x}_{t} ; \bar{\gamma}_{t}\right)\right\}$ is maximal if it is feasible, and for no other program $\left\{\left(x_{t} ; \gamma_{t}\right)\right\}$ does $\left\{c_{t}{ }^{i}\right\}$ catch up to $\left\{\bar{c}_{t}{ }^{i}\right\}$ for each $i$. Notice that this definition coincides with the usual definition of Pareto optimality when $\beta<1$. A feasible program $\left\{\left(x_{t} ; \gamma_{t}\right)\right\}$ is called envy-free if, for every $i$ and $j$, there exists $T_{0}>0$ such that $\sum_{t=1}^{T} \beta^{t-1}\left[u_{i}\left(c_{t}{ }^{i}\right)-u_{i}\left(c_{t}{ }^{j}\right)\right] \geqslant 0$ whenever $T \geqslant T_{0}$.

\section{Uniqueness of Maximal Programs}

The purpose of this section is to show that for every distribution of limiting consumption there is at most one maximal program. To do this we restrict 
attention to programs satisfying a condition necessary for maximality. First, properties of feasible programs are deduced.

Let $x_{m}=\min \left(x_{0}, \bar{x}\right)$. For $c \in\left[0, f\left(x_{m}\right)-x_{m}\right)$ define the function $g_{c}$ by $g_{c}(y)=f(y)-c$. Since $g_{c}(\bar{x})=f(\bar{x})-c>f(\bar{x})-(f(\bar{x})-\bar{x})$ and $g_{c}(\hat{x})=$ $f(\hat{x})-c \leqslant \hat{x}$, there is a $\hat{y} \in(\bar{x}, \hat{x}]$ satisfying $g_{c}(\hat{y})=\hat{y}$. Furthermore, $g_{c}(y)-y>0$ for $y \in\left[x_{m}, \hat{y}\right)$ and $g_{c}(y)-y<0$ for $y>\hat{y}$.

Lemma 2.1. The sequence defined by $y_{t}=g_{c}\left(y_{t-1}\right), y_{0}=x_{0}$ converges monotonically to $\hat{y}$.

Proof. If $y_{t} \in\left[x_{m}, \hat{y}\right)$ then

$$
\hat{y}=g_{c}(\hat{y})>g_{c}\left(y_{t}\right)=y_{t+\mathbf{1}}>y_{t} \geqslant x_{m} .
$$

Therefore, since $y_{0} \geqslant x_{m}, \hat{y}>y_{0}$ implies $y_{t}$ increases to some $\tilde{y} \in\left(x_{m}, \hat{y}\right]$. Furthermore, $\tilde{y}=g_{c}(\tilde{y})$ so $\tilde{y}=\hat{y}$. If $y_{0}>\hat{y}$ a similar argument shows that $y_{t}$ decreases to $\hat{y}$.

Lemma 2.1 can be used to establish the boundedness of feasible programs.

LeMma 2.2. Let $x_{M}=\max \left(x_{0}, \hat{x}\right)$. For any feasible program $\left\{\left(x_{t} ; \gamma_{t}\right)\right\}$, $x_{t}, c_{t}{ }^{i} \leqslant x_{M}$ for all $i$ and $t$.

Proof. Let $y_{t}=f\left(y_{t-1}\right), y_{0}=x_{0}$. By Lemma 2.1, $y_{t}$ converges monotonically to $\hat{x}$, and hence $y_{t} \leqslant x_{M}$ for all $t$. Also, for any feasible program $x_{t} \leqslant y_{t}$ for all $t$. This follows by induction since $x_{0}=y_{0}$ and if $x_{s} \leqslant y_{s}$ for some $s$, then

$$
x_{s+1}=f\left(x_{s}\right)-c_{s+1} \leqslant f\left(x_{s}\right) \leqslant f\left(y_{s}\right)=y_{s+1} .
$$

Therefore, $x_{t} \leqslant y_{t} \leqslant x_{M}$ for $t \geqslant 0$. Since $c_{t} \leqslant f\left(x_{t-1}\right)$ by feasibility, it follows that

$$
c_{t}{ }^{i} \leqslant c_{t} \leqslant f\left(x_{t-1}\right) \leqslant f\left(x_{M}\right) \leqslant x_{M}
$$

and the lemma is established.

LEMMA 2.3. Let $\left\{\left(x_{t} ; \gamma_{t}\right)\right\}$ be a feasible program and let $c \in\left(0, f\left(x_{m}\right)-x_{m}\right)$. Suppose $x_{t} \leqslant \bar{x}$ for all $t$. Then there is an such that $c_{s} \geqslant c$.

Proof. Let $y_{0}=x_{0}, y_{t}=g_{c}\left(y_{t-1}\right)$. Pick $T$ so that $y_{T}>\bar{x}$. This is possible by Lemma 2.1. Suppose $c_{t} \leqslant c$ for $t \geqslant 1$. Since

$$
x_{t}=f\left(x_{t-1}\right)-c_{t} \geqslant f\left(x_{t-1}\right)-c
$$

it follows, by induction, that $x_{t} \geqslant y_{t}$ for $t \geqslant 0$. In particular, $x_{T} \geqslant y_{T}>\bar{x}$, contradicting $x_{T} \leqslant \bar{x}$. Hence $c_{s}>c$ for somc $s$. 
The idea of the proof of the uniqueness theorem is to exploit two necessary conditions. The first, given in the following lemma, is just the usual relationship between an individual's intertemporal marginal rates of substitution. It will be used to show that maximal programs converge. That knowledge will lead us to a boundary condition relating the marginal utilities of different agents.

Lemma 2.4. Let $\left\{\left(x_{t} ; \gamma_{t}\right)\right\}$ be a maximal program. Then, for every $i$ and $s$ :

(1) If $c_{s}{ }^{i}>0$, then $u_{i}^{\prime}\left(c_{s}{ }^{i}\right) / u_{i}^{\prime}\left(c_{s+1}^{i}\right) \geqslant \beta f^{\prime}\left(x_{s}\right)$.

(2) If $c_{s+1}^{i}>0$, then $u_{i}^{\prime}\left(c_{s}^{i}\right) / u_{i}^{\prime}\left(c_{s+1}^{i}\right) \leqslant \beta f^{\prime}\left(x_{s}\right)$.

In particular, if $c_{s}^{i}, c_{s+1}^{i}>0$ then $u_{i}^{\prime}\left(c_{s}^{i}\right) / u_{i}^{\prime}\left(c_{s+1}^{i}\right)=\beta f^{\prime}\left(x_{s}\right){ }^{1}$

Proof. For fixed $i$ and $s$ define the function $h_{i}$ by

$$
h_{i}(\delta)-\beta^{s-1}\left[u_{i}\left(c_{s}{ }^{i}-\delta\right)+\beta u_{i}\left(c_{s+1}^{i}+f\left(x_{s}+\delta\right)-f\left(x_{s}\right)\right)\right] .
$$

$h_{i}(\delta)$ is the utility agent $i$ receives from consumption of $c_{s}{ }^{i}-\delta$ in period $s$ in period $s$ and $c_{s+1}^{i}+f\left(x_{s}+\delta\right)-f\left(x_{s}\right)$ in period $s+1$. Suppose $c_{s}{ }^{i}>0$, then we claim $h_{i}^{\prime}(0) \leqslant 0$. Otherwise $h_{i}(\delta)>h_{i}(0)$ for some $\delta \in\left(0, c_{s}{ }^{i}\right)$. But then the program identical with $\left\{\left(x_{t} ; \gamma_{t}\right)\right\}$ except that agent $i$ consumes $c_{s}{ }^{i}+\delta$ in period $s$ and $c_{s+1}^{i}+f\left(x_{s}+\delta\right)-f\left(x_{s}\right)$ in period $s+1$ would dominate $\left\{\left(x_{t} ; \gamma_{t}\right)\right\}$. This contradicts the maximality of $\left\{\left(x_{t} ; \gamma_{t}\right)\right\}$, so we may conclude that $c_{s}{ }^{i}>0$ implies $h_{i}^{\prime}(0) \leqslant 0$. Similarly, if $c_{s+1}^{i}>0$ then $h_{i}^{\prime}(0) \geqslant 0$. The lemma follows since

$$
h_{i}^{\prime}(0)=-\beta^{s-1}\left[u_{i}^{\prime}\left(c_{s}^{i}\right)-\beta f^{\prime}\left(x_{s}\right) u_{i}^{\prime}\left(c_{s+1}^{i}\right)\right] .
$$

The feasible program $\left\{\left(x_{t} ; \gamma_{t}\right)\right\}$ is called admissible if it satisfies conditions (1) and (2) above, for every $i$ and $t$. By Lemma 2.4 we can restrict attention to admissible programs. Our next result guarantees that admissible programs converge.

Proposition 2.5. Let $\left\{\left(x_{t} ; \gamma_{t}\right)\right\}$ be an admissible program. Then $\lim _{t \rightarrow \infty}\left(x_{t} ; \gamma_{t}\right)$ exists and is equal to $(x ; 0)$ or $(x ; \gamma)$ where $\gamma=\left(\bar{c}^{1}, \ldots, \bar{c}^{n}\right)$ and $\sum_{i=1}^{n} \bar{c}^{i}=\bar{c}$.

${ }^{1}$ Lemma 2.4 makes it clear why a common discount rate is required. Suppose that agent $i$ had a discount rate $\beta_{i}$ for $i=1$, 2. If $\beta_{1}>\beta_{2} c_{t}{ }^{i}>0$ for $t \geqslant T, i=1,2$, then Lemma 2.4 implies, for $N \geqslant 0$,

$$
\frac{u_{1}^{\prime}\left(c_{T}^{1}\right)}{u_{2}^{\prime}\left(c_{T}^{2}\right)}\left(\frac{\beta_{2}}{\beta_{1}}\right)^{N}=\frac{u_{1}^{\prime}\left(c_{T+N}^{1}\right)}{u_{2}^{\prime}\left(c_{T+N}^{2}\right)} .
$$

Hence, $\lim _{t \rightarrow \infty} c_{t}{ }^{2}=0$ and so no program satisfying (1) and (2) of Lemma 2.4 can give positive consumption to both agents in the limit. 
Proof. The proof hinges on the fact that every admissible program is eventually monotone. Indeed, we claim that

(2.1) If for some $s, x_{s-1} \geqslant x_{s}, \bar{x} \geqslant x_{s}$, then $x_{t} \geqslant x_{t+1}$ for $t \geqslant s$.

(2.2) If for some $s, x_{s-1} \leqslant x_{s}, \bar{x} \leqslant x_{s}$, then $x_{t} \leqslant x_{t+1}$ for $t \geqslant s$.

It follows from Lemma 2.4 and the concavity of $u_{i}$ that $\bar{x} \geqslant x_{x}$ implies $c_{s+1}^{i} \geqslant c_{s}^{i}$ for each $i$. Therefore,

$$
c_{s+1}=\sum_{i=1}^{n} c_{s+1}^{i} \geqslant \sum_{i=1}^{n} c_{s}^{i}=c_{s}
$$

and so if $x_{s-1} \geqslant x_{s}$, then

$$
x_{s+1}=f\left(x_{s}\right)-c_{s+1} \leqslant f\left(x_{s-1}\right)-c_{s}=x_{s} .
$$

Hence if $x_{s-1} \geqslant x_{s}$ and $\bar{x} \geqslant x_{s}$ for some $s, \bar{x} \geqslant x_{s} \geqslant x_{s+1}$. Repeated applications of this reasoning establish (2.1). A symmetric argument yields (2.2).

(2.1) and (2.2) guarantee that $\left\{\left(x_{t} ; \gamma_{t}\right)\right\}$ is eventually monotone, and, since $x_{t}$ and $\gamma_{t}$ remain bounded, it follows that $\lim _{t \rightarrow \infty}\left(x_{t} ; \gamma_{t}\right)$ exists. Let $\lim _{t \rightarrow \infty}\left(x_{t} ; \gamma_{t}\right)=(\tilde{x} ; \tilde{\gamma})$, Suppose $\tilde{x}>\bar{x}$. Then we claim $\tilde{\gamma}=0$. Otherwise $\lim _{t \rightarrow \infty} c_{t}^{i}=\tilde{c}^{i}>0$ for some $i$. Pick $T$ so that $\beta f^{\prime}\left(x_{t}\right) \leqslant 1-\delta$ for $t \geqslant T$ and some $\delta>0$. Then $c_{T}^{i} \geqslant c_{T+N}^{i} \geqslant \tilde{c}^{i}$ for $N \geqslant 0$ and, by Lemma 2.4, $\left.u_{i}^{\prime}\left(c_{T}^{i}\right)\right) u_{i}^{\prime}\left(c_{T+N}^{i}\right)=\left[\beta f^{\prime}\left(x_{T}\right)\right] \cdots\left[\beta f^{\prime}\left(x_{T+N-1}\right)\right] \leqslant(1-\delta)^{N}$. Thus $\lim _{N \rightarrow \infty} \times$ $u_{i}^{\prime}\left(c_{T}^{i}\right) / u_{i}^{\prime}\left(c_{T+N}^{i}\right)=0$. But this contradicts

$$
\frac{u_{i}^{\prime}\left(c_{T}{ }^{i}\right)}{u_{i}^{\prime}\left(c_{r+N}^{i}\right)} \geqslant \frac{u_{i}^{\prime}\left(x_{M}\right)}{u_{i}^{\prime}\left(\tilde{c}^{i}\right)}>0
$$

Hence $\tilde{\gamma}=0$ whenever $\tilde{x}>x$. Therefore,

$$
0=\lim _{t \rightarrow \infty} c_{t}=\lim _{t \rightarrow \infty}\left[f\left(x_{t-1}\right)-x_{t}\right]=f(\tilde{x})-\tilde{x} \quad \text { and so } \quad \tilde{x}=\hat{x} .
$$

To complete the proof it suffices to show that $\tilde{x}<\bar{x}$ is impossible. In order to get a contradiction, assume $\tilde{x}<\bar{x}$. Let $q$ be chosen so that $\beta f^{\prime}\left(x_{t}\right) \geqslant 1+\delta$ for all $t \geqslant q$ and some $\delta>0$. If $x_{t} \leqslant \bar{x}$ for $t \geqslant 0$, then $\left\{\gamma_{t}\right\}$ is nondecreasing and, by Lemma 2.3, there exists $c>0$ and an $r$ such that $c_{r}>c$ and so $c_{t} \geqslant c$ for $t \geqslant r$. On the other hand, if $x_{t}>\bar{x}$ for some $t$, then by (2.2) there exists $s$ such that $x_{s-1}>\bar{x} \geqslant x_{s}$ and $x_{s} \geqslant x_{t}$ for $t \geqslant s$.

In this case, for $t \geqslant s$,

$$
c_{t} \geqslant c_{s}=f\left(x_{s-1}\right)-x_{s} \geqslant f(\bar{x})-\bar{x}=\bar{c} .
$$


Now, let $T=\max (q, r, s)$ and $\epsilon=\min (\bar{c}, c) / n$. Then, for some $j, c_{t}{ }^{j}>\epsilon$ whenever $t \geqslant T$. Hence $x_{M} \geqslant c_{t}{ }^{i}$ for all $i$ and $t$ implies that, for $N \geqslant 1$,

$$
\infty>\frac{u_{j}^{\prime}(\epsilon)}{u_{j}^{\prime}\left(x_{M}\right)} \geqslant \frac{u_{j}^{\prime}\left(c_{t}^{j}\right)}{u_{j}^{\prime}\left(c_{T+N}^{j}\right)}=\left[\beta f^{\prime}\left(x_{T}\right)\right] \cdots\left[\beta f^{\prime}\left(x_{T+N-1}\right)\right] \geqslant(1+\delta)^{N} .
$$

This is impossible, so $\tilde{x}<\hat{x}$ is ruled out. The observation that if $\lim _{t \rightarrow \infty} x_{t}=\bar{x}$ then $\lim _{t \rightarrow \infty} c_{t}=f(\bar{x})-\bar{x}=\bar{c}$ completes the proof.

We have now restricted possible efficient programs to those that converge. Proposition 2.6 guarantees that the limiting consumption will be positive.

Proposition 2.6. Suppose $\left\{\left(x_{t} ; \gamma_{t}\right)\right\}$ is maximal. Then $\lim _{t \rightarrow \infty} x_{t}=\bar{x}$.

Proof. Suppose the proposition is false. Let $\left\{\left(x_{t} ; \gamma_{t}\right)\right\}$ be a maximal program such that $\lim _{t \rightarrow \infty} x_{t}=\hat{x}$. Choose $T$ so that $x_{t} \geqslant \bar{x},\left|\hat{x}-x_{t}\right|<\bar{c} / 4$, and $\left|\hat{x}-f\left(x_{t}\right)\right|<\bar{c} / 4$ whenever $t \geqslant T$. It follows that

$$
c_{t}=f\left(x_{t-1}\right)-x_{t} \leqslant\left|f\left(x_{t-1}\right)-\hat{x}\right|+\left|\hat{x}-x_{t}\right|<\bar{c} / 2 .
$$

Consider the program $\left\{\left(\tilde{x}_{t} ; \tilde{\gamma}_{t}\right)\right\}$ where

$$
\begin{aligned}
& \left(\tilde{x}_{t} ; \tilde{\gamma}_{t}\right)=\left(x_{t} ; \gamma_{t}\right) \quad \text { if } t<T \text {, } \\
& \tilde{x}_{t}=\bar{x} \quad \text { if } t \geqslant T \text {, and } \\
& \tilde{c}_{t}= \begin{cases}f\left(x_{T-1}\right)-\bar{x} & \text { if } t=T \\
\bar{c} & \text { if } t>T .\end{cases}
\end{aligned}
$$

Since $\tilde{c}_{t}>c_{t}$ for $t>T$ we can choose $\tilde{\gamma}_{t}=\left(\tilde{c}_{t}{ }^{1}, \ldots, \tilde{c}_{t}{ }^{n}\right)$ such that $\sum_{i=1}^{n} \tilde{c}_{t}{ }^{i}=\tilde{c}_{t}$ and $\tilde{c}_{t}{ }^{i}>c_{t}{ }^{i}$ for every $i$. Therefore $\left\{\left(\tilde{x}_{t} ; \tilde{\gamma}_{t}\right)\right\}$ dominates $\left\{\left(x_{t} ; \gamma_{t}\right)\right\}$, contradicting maximality.

Taken together, the last two results demonstrate that all maximal programs are globally asymptotically stable.

Proposition 2.7. Suppose $\left\{\left(x_{t} ; \gamma_{t}\right)\right\}$ is an admissible program such that $\lim _{t \rightarrow \infty} x_{t}=\bar{x}$. Then $\left\{\left(x_{t} ; \gamma_{t}\right)\right\}$ is a monotone sequence, increasing if $x_{o}<\bar{x}$, constant if $x_{o}=\bar{x}$, and decreasing if $x_{0}>\bar{x}$.

Proof. The proposition follows immediately from (2.1) and (2.2).

In order to show that a given distribution of limiting consumption can correspond to no more than one maximal program we must relate period $t$ consumption to limiting consumption. In order to do this we introduce 
$M_{i j}=u_{i}^{\prime}\left(\theta_{i} \bar{c}\right) / u_{j}^{\prime}\left(\theta_{j} \bar{c}\right)$ for $1 \leqslant i, j \leqslant n$. Clearly $0<M_{i j}<\infty$ and $M_{i j} M_{j k}=M_{i k}$. The fact that the limiting ratio of marginal utilities is related to the ratios in period $t$ will guarantee that no two maximal programs will yield the same limiting consumption.

If $\left\{\left(x_{t} ; \gamma_{t}\right)\right\}$ is an admissible program with $\lim _{t \rightarrow \infty}\left(x_{t} ; \gamma_{t}\right)=(\bar{x} ; \bar{c} \theta)$ then

(2.3) $\beta f^{\prime}\left(x_{t}\right) \geqslant u_{j}^{\prime}\left(c_{t}^{j}\right) / u_{j}^{\prime}\left(c_{t+1}^{j}\right)$ for all $j$ and $t$

with equality whenever $c_{t}{ }^{j}>0$.

(2.3) is a consequence of Proposition 2.7 and Lemma 2.4 unless $0=c_{t}{ }^{j}=$ $c_{t+1}^{j}$. But if $c_{t}{ }^{j}=c_{t+1}^{j}=0$ then $\beta f^{\prime}\left(x_{t}\right) \geqslant 1=u_{j}^{\prime}(0) / u_{j}^{\prime}(0)$. Therefore if $c_{t}^{i}>0$,

$$
\frac{u_{i}^{\prime}\left(c_{t}{ }^{i}\right)}{u_{i}^{\prime}\left(c_{t+N}^{i}\right)}=\left[\beta f^{\prime}\left(x_{t}\right)\right] \cdots\left[\beta f^{\prime}\left(x_{t+N-1}\right)\right] \geqslant \frac{u_{j}^{\prime}\left(c_{t}{ }^{j}\right)}{u_{j}^{\prime}\left(c_{t+N}^{j}\right)} \quad \text { for } \quad N \geqslant 1 .
$$

Hence, taking limits, we may conclude

(2.4) $u_{i}^{\prime}\left(c_{t}{ }^{i}\right) / u_{j}^{\prime}\left(c_{t}^{j}\right) \geqslant M_{i j} \quad$ whenever $c_{t}^{i}>0$.

(2.4) is a necessary condition for optimality. In order to show uniqueness, we need to show that there is only one solution $\gamma=\left(c^{1}, \ldots, c^{n}\right)$ to (2.4) satisfying $\sum_{i=1}^{n} c^{i}=c$.

LeMmA 2.8. Suppose $\left(c^{1}, \ldots, c^{n}\right)$ and $\left(\tilde{c}^{1}, \ldots, \tilde{c}^{n}\right)$ satisfy (2.4). If $c^{j}>\tilde{c}^{j}$ for some $j$, then $c^{i}>\tilde{c}^{i}$ whenever $\tilde{c}^{i}>0$.

Proof. Suppose $c^{j}>\tilde{c}^{j}$ and $\tilde{c}^{k}>0$. We have

$$
\frac{u_{j}^{\prime}\left(c^{j}\right)}{u_{k}^{\prime}\left(c^{k}\right)} \geqslant M_{j k} \quad \text { and } \quad \frac{u_{k}^{\prime}\left(\tilde{c}^{k}\right)}{u_{j}^{\prime}\left(\tilde{c}^{j}\right)} \geqslant M_{k, j}
$$

hence,

$$
\frac{u_{j}^{\prime}\left(c^{j}\right)}{u_{k}^{\prime}\left(c^{k}\right)} \geqslant \frac{u_{j}^{\prime}\left(\tilde{c}^{j}\right)}{u_{k}^{\prime}\left(\tilde{c}^{1}\right)}
$$

and so

$$
1>\frac{u_{j}^{\prime}\left(c^{j}\right)}{u_{j}^{\prime}\left(\tilde{c}^{j}\right)} \geqslant \frac{u_{k}^{\prime}\left(c^{k}\right)}{u_{k}^{\prime}\left(\tilde{c}^{k}\right)}
$$

and thus $c^{k}>\tilde{c}^{k}$.

We now are prepared to prove the main result of this section.

THEOREM 2.9. There is at most one maximal program $\left\{\left(x_{t} ; \gamma_{t}\right)\right\}$ starting from $x_{0}$ such that $\lim _{t \rightarrow \infty} \gamma_{t}=\bar{c} \theta$.

Proof. Suppose $\left\{\left(x_{t} ; \gamma_{t}\right)\right\}$ and $\left\{\left(\tilde{x}_{t} ; \tilde{y}_{t}\right)\right\}$ are two different programs such that $\lim _{t \rightarrow \infty}\left(x_{i} ; \gamma_{t}\right)=\lim _{t \rightarrow \infty}\left(\tilde{x}_{t} ; \tilde{\gamma}_{t}\right)=(\bar{x} ; \bar{c} \theta)$. Then there is an $s \geqslant 1$ 
such that $\left(x_{t} ; \gamma_{t}\right)=\left(\tilde{x}_{t} ; \tilde{\gamma}_{t}\right)$ for $t \leqslant s$, and $\gamma_{s+1} \neq \tilde{\gamma}_{s+1}$. Without loss of generality, assume $\sum_{i=1}^{n} c_{s+1}^{i}>\sum_{i=1}^{n} \tilde{c}_{s+1}^{i}$. Then we claim that for all $t>s$

(2.5) $x_{t}<\tilde{x}_{t}$ and $c_{t}^{i}>\tilde{c}_{t}^{i}$ whenever $c_{t}^{i}>0$.

This follows because $\sum_{i=1}^{n} c_{s+1}^{i}>\sum_{i=1}^{n} \tilde{c}_{s+1}^{i}$ and

$$
x_{s+1}=f\left(x_{s}\right)-\sum_{i=1}^{n} c_{s+1}^{i}<f\left(\tilde{x}_{s}\right)-\sum_{i=1}^{n} \tilde{c}_{s+1}^{i}=\tilde{x}_{s+1} .
$$

But both $\gamma_{s+1}$ and $\tilde{\gamma}_{s+1}$ satisfy (2.4), so it follows from Lemma 2.8 that $c_{s+1}^{i}>\hat{c}_{s+1}^{i}$ whenever $\tilde{c}_{s+1}^{i}>0$. This establishes (2.5) when $t=s+1$. Now if (2.5) holds for some $T>s$ we may apply (2.3) and $x_{T}<\tilde{x}_{T}$ to conclude, provided $c_{T}^{i}>0$,

$$
\frac{u_{i}^{\prime}\left(c_{T}^{i}\right)}{u_{i}^{\prime}\left(c_{T+1}^{i}\right)}=\beta f^{\prime}\left(x_{T}\right)>\beta f^{\prime}\left(\tilde{x}_{T}\right) \geqslant \frac{u_{i}^{\prime}\left(\tilde{c}_{T}^{i}\right)}{u_{i}^{\prime}\left(\tilde{c}_{T+1}^{i}\right)} .
$$

Hence, since $c_{T}{ }^{i} \geqslant \tilde{c}_{T}{ }^{i}, c_{T}{ }^{i}>0$ implies $c_{T+1}^{i}>\tilde{c}_{T+1}^{i}$. Moreover, by Proposition 2.7, $c_{T}^{j}>0$ for some $j$, so $c_{T+1}^{i}>\tilde{\epsilon}_{T+1}^{i}$ and therefore, since $\tilde{\gamma}_{T+1}$ and $\gamma_{T+1}$ both must satisfy (2.4), it follows from Lemma 2.8 that $c_{T+1}^{i}>\tilde{c}_{T+1}^{i}$ whenever $\tilde{c}_{T+1}^{i}>0$. Hence

$$
\sum_{i=1}^{n} c_{T+1}^{i}>\sum_{i=1}^{n} \tilde{c}_{T+1}^{i}
$$

and

$$
x_{T+1}=f\left(x_{T}\right)-\sum_{i=1}^{n} c_{T+1}^{i}<f\left(\tilde{x}_{T}\right)-\sum_{i=1}^{n} \tilde{c}_{T+1}^{i}=\tilde{x}_{T+1} .
$$

Claim (2.5) then follows by induction.

Now select $T \geqslant s$ so that for $t \geqslant T, \tilde{c}_{t}{ }^{1}>0$. This is possible since $\lim _{t \rightarrow \infty} \tilde{c}_{t}{ }^{1}=\theta_{1} \bar{c}>0$. From (2.5), $c_{t}{ }^{1}>\tilde{c}_{t}{ }^{1}$ for $t \geqslant T$. Therefore, for all $N$,

$$
\frac{u_{1}^{\prime}\left(c_{T+N}^{1}\right)}{u_{1}^{\prime}\left(\tilde{c}_{T+N}^{1}\right)}=\frac{u_{1}^{\prime}\left(c_{T}^{1}\right)}{u_{1}^{\prime}\left(\tilde{c}_{T}^{1}\right)}\left[\frac{\beta f^{\prime}\left(\tilde{x}_{T}\right)}{\beta f^{\prime}\left(x_{T}\right)}\right] \cdots\left[\frac{\beta f^{\prime}\left(\tilde{x}_{T+N-1}\right)}{\beta f^{\prime}\left(x_{T+N-1}\right)}\right]
$$

But $x_{t}<\tilde{x}_{t}$ for $t \geqslant T$ so, for $N \geqslant 1$,

Thus

$$
1>\frac{u_{1}^{\prime}\left(c_{T}^{1}\right)}{u_{1}^{\prime}\left(\tilde{c}_{T}^{1}\right)} \geqslant \frac{u_{1}^{\prime}\left(c_{T+N}^{1}\right)}{u_{1}^{\prime}\left(\tilde{c}_{T+N}^{1}\right)}
$$

$$
1>\lim _{N \rightarrow \infty} \frac{u_{1}^{\prime}\left(c_{T+N}^{1}\right)}{u_{1}^{\prime}\left(\tilde{c}_{T+N}^{1}\right)}=\frac{u_{1}^{\prime}\left(\theta_{1} \bar{c}\right)}{u_{1}^{\prime}\left(\theta_{1} \bar{c}\right)}=1 .
$$

This is impossible and the contradiction establishes the theorem. 


\section{Existence of Maximal Programs}

The purpose of this section is to prove that maximal programs exist. It turns out that this is an easy consequence of the existence of maximal programs in economies with a single utility-maximizing agent. The function $U$ we choose is defined by

$$
U(c)=\max \sum_{i=1}^{n} \frac{u_{i}\left(c^{i}\right)}{u_{i}^{\prime}\left(\theta_{i} \bar{c}\right)} \quad \text { subject to } \quad c^{i} \geqslant 0, \sum_{i=1}^{n} c^{i}=c .
$$

Clearly, $U$ is continuous and strictly concave. Also, since each $u_{i}$ is strictly concave, every $c \geqslant 0$ determines a unique vector $\gamma(c)=\left(c^{1}, \ldots, c^{n}\right)$ such that $c^{i} \geqslant 0, c=\sum c^{i}$, and $U(c)=\sum\left(u_{i}\left(c^{i}\right) / u_{i}^{\prime}\left(\theta_{i} \bar{c}\right)\right)$. We call $\left\{\left(x_{t}, c_{t}\right)\right\}$ a feasible sequence (from $x_{o}$ ) if $x_{t} \geqslant 0, c_{t} \geqslant 0$, and $c_{t}=f\left(x_{t-1}\right)-x_{t}$ for all $t \geqslant 1$. Thus, associated with every feasible sequence $\left\{\left(x_{t} ; c_{t}\right)\right\}$ is a feasible program $\left\{\left(x_{t} ; c_{t}\right)\right\}$ where $\gamma_{t}=\gamma\left(c_{t}\right)$.

It is not surprising that efficient allocations can be generated by an appropriately weighted sum of individual utilities. The relationships among efficient allocations, weighted averages of utilities, and competitive equilibria are well known (see, for example, [6]). In this case the situation is altered somewhat because (when $\beta=1$ ) competitive equilibria may not exist.

For reference we state

LemMA 3.1. Given any $x_{0}>0$ there is a unique feasible sequence $\left\{\left(\bar{x}_{t} ; \bar{c}_{t}\right)\right\}$ such that for any other feasible sequence

$$
\liminf _{T \rightarrow \infty} \sum_{t=1}^{T} \beta^{t}{ }^{1}\left[U\left(c_{t}\right)-U\left(\bar{c}_{t}\right)\right]<0 .
$$

Lemma 3.1 is the standard existence theorem, well known in the theory of optimal growth. Proofs can be found (for the case $\beta=1$ ) in Brock [1] or Gale [4].

We can now prove

THEOREM 3.2. There exists a maximal program $\left\{\left(\bar{x}_{t} ; \bar{\gamma}_{t}\right)\right\}$ such that $\lim _{t \rightarrow \infty} \bar{\gamma}_{t}=\theta \bar{c}$.

Proof. Let $\left\{\left(\bar{x}_{t} ; \bar{c}_{t}\right)\right\}$ be a feasible sequence guaranteed by Lemma 3.1. Let $\bar{\gamma}_{t}=\gamma\left(\bar{c}_{t}\right)$. Then $\left\{\left(\bar{x}_{t} ; \bar{\gamma}_{t}\right)\right\}$ is maximal. For suppose $\left\{\left(x_{t} ; \gamma_{t}\right)\right\}$ is a feasible program such that $\lim _{\inf _{T \rightarrow \infty}} \sum_{t=1}^{T} \beta^{t-1}\left[u_{i}\left(c_{t}{ }^{i}\right)-u_{i}\left(\bar{c}_{t}{ }^{i}\right)\right] \geqslant 0$ for each $i$. Then

$$
\begin{aligned}
\liminf _{T \rightarrow \infty} & \sum_{t=1}^{T} \beta^{t-1}\left[U\left(c_{t}\right)-U\left(\bar{c}_{t}\right)\right] \\
\quad= & \lim _{T \rightarrow \infty} \sum_{t=1}^{T} \sum_{i=1}^{n} \frac{\beta^{t-1}}{u_{i}^{\prime}\left(\theta_{i} \bar{c}\right)}\left[u_{i}\left(c_{t}{ }^{i}\right)-u_{i}\left(\bar{c}_{t}{ }^{i}\right)\right] \\
& \geqslant \sum_{i=1}^{n} \liminf _{T \rightarrow \infty} \sum_{t=1}^{T} \frac{\beta^{t-1}}{u_{i}^{\prime}\left(\theta_{i} \bar{c}\right)}\left[u_{i}\left(c_{t}{ }^{i}\right)-u_{i}\left(\bar{c}_{t}{ }^{i}\right)\right] \geqslant 0 .
\end{aligned}
$$


But, by Lemma 3.1, this can only happen if $\left\{\left(x_{t} ; \gamma_{t}\right)\right\}=\left\{\left(\bar{x}_{\ell} ; \bar{\gamma}_{t}\right)\right\}$. Hence $\left\{\left(\bar{x}_{t} ; \bar{\gamma}_{t}\right)\right\}$ is maximal.

It remains to show that $\lim _{t \rightarrow \infty} \gamma_{t}=\theta \bar{c}$. Because $\left\{\left(\bar{x}_{t} ; \bar{\gamma}_{t}\right)\right\}$ is maximal, it follows from Proposition 2.5 that $\lim _{t \rightarrow \infty} \gamma_{t}$ exists. Denote this limit by $\left(\bar{c}_{1}, \ldots, \bar{c}_{n}\right)$. But, by Propositions 2.5 and $2.6, \sum_{i=1}^{n} \bar{c}_{i}=\bar{c}$ and so $\left(\bar{c}_{1}, \ldots, \bar{c}_{n}\right)$ solve:

$$
\text { Maximize } \sum_{i=1}^{n} \frac{u_{i}\left(c_{i}\right)}{u_{i}^{\prime}\left(\theta_{i} \bar{c}\right)} \text { subject to } \sum_{i=1}^{n} c_{i}=\bar{c}, \quad c_{i} \geqslant 0 .
$$

Therefore, $u_{i}^{\prime}\left(\bar{c}_{i}\right) / u^{\prime}\left(\theta_{i} \bar{c}\right) \geqslant u_{j}^{\prime}\left(\bar{c}_{j}\right) / u_{j}^{\prime}\left(\theta_{j} \bar{c}\right)$ whenever $\bar{c}_{i}>0$. Thus, $\sum_{i=1}^{n} \bar{c}_{i}=\bar{c}$ and $u_{i}^{\prime}\left(\bar{c}_{i}\right) / u_{j}^{\prime}\left(\bar{c}_{j}\right) \geqslant M_{i j}$ whenever $\bar{c}_{i}>0$ and we must have $\bar{c}_{i}=\theta_{i} \bar{c}$ by Lemma 2.8. It follows that $\lim _{t \rightarrow \infty} \gamma_{t}=\theta \bar{c}$, completing the proof.

Combined with Theorem 2.9, this result guarantees the existence of a unique maximal program associated with every distribution of limiting consumptions.

\section{Fair Allocations}

The results of Sections II and III show that there is a unique maximal program corresponding to every limiting distribution of consumption. This section discusses the properties of maximal programs, with emphasis on that program which gives equal shares to each agent in the limit.

Throughout this section we assume $\beta=1$. It is necessary to distinguish between the $\beta<1$ and the Ramsey case because when $\beta<1$ the utility stream received by an agent is finite and the fact that an agent's envy is finite is of no intcrest. Furthermore, the maximal allocation with equal limiting shares of consumption need not be free from envy (as Theorem 4.5 shows) while in the $\beta<1$ case the envy-free allocations exist by analogy to finite horizon models.

LEMMA 4.1. For every maximal program $\left\{\left(\bar{x}_{t} ; \bar{\gamma}_{t}\right)\right\}, \sum_{t=1}^{\infty}\left|\bar{x}_{t}-\bar{x}\right|<\infty$.

Proof. If $x_{0}<\bar{x}$, Proposition 2.7 guarantees that $x_{0} \leqslant x_{t} \leqslant \bar{x}$ for all $t$. Hence, by Lemma 2.3, there is a $T$, a $c>0$, and a $j$ such that $c_{t}{ }^{j}>c$ for $t \geqslant T$. It follows from Lemma 2.4 that

$$
\frac{u_{j}^{\prime}(c)}{u_{j}^{\prime}\left(x_{M}\right)} \geqslant \frac{u_{j}^{\prime}\left(\bar{c}_{t}^{j}\right)}{u_{j}^{\prime}\left(\bar{c}_{t+N}^{j}\right)}=f^{\prime}\left(\bar{x}_{t}\right) \cdots f^{\prime}\left(\bar{x}_{t+N-1}\right) \quad \text { for } \quad N \geqslant 1 .
$$

Therefore, $u_{j}^{\prime}(c) / u_{j}^{\prime}\left(x_{M}\right) \geqslant \prod_{t \geqslant N} f^{\prime}\left(x_{t}\right)$ and so $\prod_{t=1}^{\infty} f^{\prime}\left(\bar{x}_{t}\right)<\infty$. It is well known that this implies $\sum_{t=1}^{\infty}\left[f^{\prime}\left(\bar{x}_{t}\right)-1\right]<\infty$; hence, since $f^{\prime}(\bar{x})=1$ we have $\infty>\sum_{t=1}^{\infty}\left[f^{\prime}\left(\bar{x}_{t}\right)-f^{\prime}(\bar{x})\right] \geqslant m \sum_{t=1}^{\infty}\left(\bar{x}-\bar{x}_{t}\right)$ where $m=$ 
$\min _{\xi \in\left[x_{0}, x\right]}\left(-f^{\prime \prime}(\xi)\right)>0$. This proves the lemma when $x_{o}<\bar{x}$. A similar argument establishes the lemma when $x_{0}>\bar{x}$.

THEOREM 4.2. For any maximal program $\left\{\left(\bar{x}_{t} ; \bar{\gamma}_{t}\right)\right\}, \sum_{t=1}^{\infty}\left|\bar{c}-\bar{c}_{t}\right|<\infty$.

Proof. Since $\bar{c}_{t}=f\left(\bar{x}_{t-1}\right)-\bar{x}_{t}$ and $f(x)-x \leqslant \bar{c}$ for all $x \geqslant 0$ we have

$$
\sum_{t=1}^{T}\left(\bar{c}_{t}-\bar{c}\right)=\sum_{t=1}^{T}\left[\left(f\left(\bar{x}_{t}\right)-\bar{x}_{t}\right)-\bar{c}\right]+f\left(x_{o}\right)-f\left(\bar{x}_{T}\right)<f\left(x_{o}\right) .
$$

This establishes the theorem when $x_{0} \geqslant \bar{x}$ for in that case $\vec{c}_{t} \geqslant \bar{c}$ for all $t$.

If $x_{n}<\bar{x}$, then $\bar{c}>\bar{c}_{t}$ for all $t$ and

$$
\begin{aligned}
\bar{c}-\bar{c}_{t} & =(f(\bar{x})-\bar{x})-\left(f\left(\bar{x}_{t-1}\right)-\bar{x}_{t}\right) \\
& =\left(f(\bar{x})-f\left(\bar{x}_{t-1}\right)\right)+\left(\bar{x}_{t}-\bar{x}_{t-1}\right)-\left(\bar{x}-\bar{x}_{t-1}\right) \\
& \leqslant\left(\bar{x}-\bar{x}_{t-1}\right) f^{\prime}\left(x_{o}\right)+\left(\bar{x}_{t}-\bar{x}_{t-1}\right)-\left(\bar{x}-\bar{x}_{t-1}\right) \\
& =\left(\bar{x}-\bar{x}_{t-1}\right)\left(f^{\prime}\left(x_{o}\right)-1\right)+\left(\bar{x}_{t}-\bar{x}_{t-1}\right) .
\end{aligned}
$$

Hence $\sum_{t=1}^{\infty}\left(\bar{c}-\bar{c}_{t}\right)=\left(f^{\prime}\left(x_{0}\right)-1\right) \sum_{t=1}^{\infty}\left(\bar{x}-\bar{x}_{t-1}\right)+\sum_{t=1}^{\infty}\left(\bar{x}_{t}-\bar{x}_{t-1}\right)$. This completes the proof since $\sum_{t=1}^{\infty}\left(\bar{x}-\bar{x}_{t-1}\right)<\infty$ by Lemma 4.1 and $\sum_{t=1}^{\infty}\left(x_{t}-x_{t-1}\right)=\bar{x}-x_{0}$.

Theorem 4.2 says that no program can yield infinitely more consumption than a maximal program. Corollary 4.3 makes an analogous statement about utilities.

CoRollary 4.3. Suppose $\left\{\left(\bar{x}_{t} ; \bar{\gamma}_{t}\right)\right\}$ is a maximal program. If $\lim _{t \rightarrow \infty} \gamma_{t}=\bar{c} \theta$, then $\sum_{t=1}^{\infty}\left|\bar{c}_{t}{ }^{i}-\theta_{i} \bar{c}\right|<\infty$ and $\sum_{t=1}^{\infty}\left|u_{i}\left(\theta_{i} \bar{c}\right)-u_{i}\left(\bar{c}_{t}{ }^{i}\right)\right|<\infty$.

Proof. By Proposition 2.7, $\left|\bar{c}_{t}^{i}-\theta_{i} \bar{c}\right| \leqslant\left|\bar{c}_{t}-\bar{c}\right|$ for all $i$ and $t$. So $\sum_{t=1}^{\infty}\left|\bar{c}_{t}{ }^{i}-\theta_{i} \bar{c}\right|<\infty$ by Theorem 4.2. Also

$$
\left|u_{i}\left(\bar{c}_{t}^{i}\right)-u_{i}\left(\theta_{i} \bar{c}\right)\right|=u_{i}^{\prime}\left(\xi_{t}\right)\left(\left|\bar{c}_{t}^{i}-\theta_{i} \bar{c}\right|\right) \leqslant M_{i}\left(\left|\bar{c}_{t}-\bar{c}\right|\right)
$$

where $M_{i}=\max \left(u_{i}^{\prime}\left(\bar{c}_{1}\right), u_{i}^{\prime}\left(\theta_{i} \bar{c}\right)\right)$. The corollary now follows from another application of Theorem 4.2.

The next result guarantees that the maximal program giving each agent limiting consumption $\bar{c} / n$ is almost envy-free, in that each individual receives - up to a finite amount - as much utility from his consumption sequence as from that of any one else. Clearly, no other maximal allocation will have this property; with any other limiting consumptions there would be a $T$ and an $\epsilon>0$ such that, for some $i$ and $j$ and all $t \geqslant T$,

$$
\bar{c}_{t}{ }^{i}>\bar{c} / n+\epsilon>\bar{c} / n-\epsilon>\bar{c}_{t}{ }^{j} .
$$


Agent $j$ would then prefer $i$ 's consumption to his own by an infinite amount.

Corollary 4.4. If $\theta_{i}=1 / n$ for each $i$, then

$$
\sum_{t=1}^{\infty}\left|\bar{c}_{t}{ }^{i}-\bar{c}_{t}{ }^{j}\right|<\infty \quad \text { and } \quad \sum_{t-1}^{\infty}\left|u_{i}\left(\bar{c}_{t}{ }^{i}\right)-u_{i}\left(\bar{c}_{t}^{j}\right)\right|<\infty
$$

for every $i$ and $j$.

Proof. Since $\left|\bar{c}_{t}{ }^{i}-\bar{c}_{t}{ }^{j}\right| \leqslant\left|\bar{c}_{t}{ }^{i}-\bar{c} / n\right|+\left|\bar{c}_{t}{ }^{j}-\bar{c} / n\right|$, the fact that $\sum_{t=1}^{\infty}\left|\bar{c}_{t}{ }^{i}-\bar{c}_{t}{ }^{j}\right|<\infty$ follows from Theorem 4.2. Also, if $M_{i}=\max \times$ $\left[u_{i}^{\prime}\left(\bar{c}_{1}{ }^{i}\right), u_{i}^{\prime}(\bar{c} / n)\right]$, then

$$
\sum_{t=1}^{\infty}\left|u_{i}\left(\bar{c}_{t}{ }^{i}\right)-u_{i}\left(\bar{c}_{t}{ }^{j}\right)\right| \leqslant M_{i} \sum_{t=1}^{\infty}\left|\bar{c}_{t}^{i}-\bar{c}_{t}^{j}\right|<\infty .
$$

Unless the agents have identical utility functions, no maximal allocation is guaranteed to be envy-free. In fact, the following theorem implies that in many circumstances there exists $i$ and $j$ such that $\bar{c}_{t}{ }^{i}>\bar{c}_{t}{ }^{j}$ for all $t$.

THEOREM 4.5. Suppose $\left\{\left(\bar{x}_{t} ; \bar{\gamma}_{t}\right)\right\}$ is a maximal program and $\lim _{t \rightarrow \infty} \bar{\gamma}_{t}=$ $\bar{c}(1 / n, \ldots, 1 / n)$. If, for some $i$ and $j, u_{i}=g \circ u_{j}$ where $g$ is continuously differentiable, increasing, and concave then

(1) If $x_{0}<\bar{x}$, then $\bar{c}_{t}{ }^{i}>\bar{c}_{t}^{j}$ whenever $\bar{c}_{t}^{j}>0$.

(2) If $x_{0}>\bar{x}$, then $\bar{c}_{i}{ }^{j}>\bar{c}_{t}{ }^{i}$ for all $t$.

Proof. Suppose $x_{v}<\bar{x}$. Then, by Proposition 2.8, $\bar{c}_{t}{ }^{j}, \bar{c}_{t}{ }^{j}<\bar{c} / n$ for all $t$. If, for some $s, \bar{c}_{s}{ }^{j}>0$ and $\bar{c}_{s}{ }^{j} \geqslant \bar{c}_{s}{ }^{i}$ then

$$
\frac{u_{j}^{\prime}(\bar{c} / n)}{u_{i}^{\prime}(\bar{c} / n)} \leqslant \frac{u_{j}^{\prime}\left(\bar{c}_{s}^{j}\right)}{u_{i}^{\prime}\left(\bar{c}_{s}^{i}\right)} \quad \text { by }(2.4)
$$

but

$$
\frac{u_{j}^{\prime}(\bar{c} / n)}{u_{i}^{\prime}(\bar{c} / n)}=\frac{u_{j}^{\prime}(\bar{c} / n)}{g^{\prime}\left(u_{j}(\bar{c} / n)\right) u_{j}^{\prime}(\bar{c} / n)}=\frac{1}{g^{\prime}\left(u_{j}(\bar{c} / n)\right)}
$$

and

$$
\frac{u_{j}^{\prime}\left(\bar{c}_{s}^{j}\right)}{u_{i}^{\prime}\left(\bar{c}_{s}^{i}\right)} \leqslant \frac{u_{j}^{\prime}\left(\bar{c}_{s}^{i}\right)}{u_{i}^{\prime}\left(\bar{c}_{s}^{i}\right)}=\frac{1}{g^{\prime}\left(u_{j}\left(\bar{c}_{s}^{i}\right)\right)}
$$

Hence $g^{\prime}\left(u_{j}\left(\bar{c}_{s}{ }^{i}\right)\right) \leqslant g^{\prime}\left(u_{j}(\bar{c} / n)\right.$ and thus $\bar{c}_{s}{ }^{i} \geqslant \bar{c} / n$, by the concavity of $g$. This contradiction establishes (1). (2) follows from a similar argument and the observation that if $x_{0}>\bar{x}$ then $c_{t}^{i}>0$ for all $t$. 
We can interpret this theorem in the following way. The more concave (risk averse) an agent's utility function, the more he prefers to have consumption restricted to a small interval. Since consumption in the maximal program is monotone, the most concave agent will receive more than the others when $x_{0}<\bar{x}$ and less when $x_{0}>\bar{x}$.

It is easy to see that unless $u_{i}=a u_{j}+b$ for some $a>0, u_{i}=g \circ u_{j}$ for some increasing $g$, where either $g$ or $g^{-1}$ is strictly concave over some interval. Therefore, provided two agents have different preferences, there is a production function and an initial stock $x_{o}$ that guarantees that some agent consumes more in every period than another agent in the maximal program with equal limiting consumptions.

\section{ACKNOWLEDGMENT}

I would like to thank David Gale for valuable assistance throughout the preparation of this paper. Thanks also go to Andreu Mas-Colell,

\section{REFERENCES}

1. W. A. Brock, On existence of weakly maximal programmes in a multisector economy Rev. Econ. Stud. 37 (1970).

2. W. A. Brock and J. Scheinkman, Global asymptotic stability of optimal control systems with application to the theory of economic growth, J. Econ. Theory 12 (1976).

3. E. Burmeister AND R. Dobell, "Mathematical Theories of Economic Growth," Macmillan, New York, 1970.

4. D. Gale, On optimal development in a multisector economy, Rev. Econ. Stud. 34 (1967).

5. D. Levhari and L. J. Mirman, "The Great Fish War: An Example Using a Dynamic Cournot-Nash Solution," University of Illinois, Urbana, March 1977.

6. H. NiKaldo, Welfare economics and evidence of an equilibrium for a competitive economy, Metroeconomica 12 (1960).

7. E. PAZner, Pitfalls in the theory of fairness, J. Econ. Theory 14 (1977).

8. E. Pazner and D. Schmeidler, A difficulty in the concept of fairness, Rev. Econ. Stud. 41 (1974).

9. J. Rawls, "A Theory of Justice," Harvard Univ. Press, Cambridge, Mass., 1971.

10. H. Varian, Equity, envy and efficiency, J. Econ. Theory 9 (1974). 